\title{
Photoproduction of neutral pions
}

\section{Andreas Fuhrer*}

Department of Physics, University of California at San Diego, La Jolla, CA 92093

E-mail: afuhrer@physics.ucsd.edu

\begin{abstract}
The pion and nucleon mass differences generate a very pronounced cusp in the photoproduction reaction of a single neutral pion on the proton. A nonrelativistic effective field theory to describe this reaction is constructed. The approach is rigorous in the sense that it is an effective field theory with a consistent power counting scheme. Expressions for the $S$ - and $P$-wave multipole amplitudes at one loop are given. The relation of the phase of the electric multipole $E_{0+}$ to the phase of the $S$-wave of $\pi^{0} p \rightarrow \pi^{0} p$ scattering is discussed.
\end{abstract}

6th International Workshop on Chiral Dynamics

July 6-10 2009

Bern, Switzerland

${ }^{*}$ Speaker. 


\section{Introduction}

The photoproduction reaction of neutral pions on the proton is a reaction which shows a strong effect due to isospin breaking. The electric multipole $E_{0+}$ exhibits an exceptionally strong cusp at the $\pi^{+} n$ threshold (see for instance Ref. [1]). The strength of this cusp is intimately related to the charge exchange scattering length of pion-nucleon scattering. Therefore, accurate experimental data of the photoproduction reaction allows one to access the pion-nucleon scattering lengths.

Along the lines of previous work which analyzed the cusps in $K \rightarrow 3 \pi$ and $\eta^{\prime} \rightarrow \eta \pi \pi$ decays [2-4], we construct a nonrelativistic theory which provides a rigorous framework to describe the structure of the cusp order by order in a perturbative expansion in terms of small momenta and effective range parameters of pion-nucleon scattering as well as threshold parameters of the photoproduction reaction [5]. By construction, the theory correctly reproduces the low-energy singularities in the Mandelstam plane.

The cusp in neutral pion photoproduction has been studied before. Ref. [6] introduces a twoparameter model, which captures the most important leading effect of the cusp. In Ref. [7], a coupled channel $S$-matrix approach is used to investigate the cusp structure.

\section{Multipole decomposition}

Some basic relations and definitions used in the analysis of pion photoproduction are collected. We calculate the matrix element for the process $p\left(p_{1}\right)+\gamma(k) \rightarrow p\left(p_{2}\right)+\pi^{0}(q)$ at leading order in the electromagnetic coupling $e$,

$$
\left.\left\langle p_{2}, q \text { out }\right| p_{1}, k \text { in }\right\rangle=-i(2 \pi)^{4} \delta^{(4)}\left(P_{f}-P_{i}\right) \bar{u}\left(p_{2}, t^{\prime}\right) \varepsilon_{\mu} J^{\mu} u\left(p_{1}, t\right),
$$

where $P_{i}$ and $P_{f}$ denote the total four momentum in the initial and in the final state, respectively and $\varepsilon^{\mu}$ stands for the polarization vector of the photon.

To analyze the photoproduction reaction of pions, electric and magnetic multipoles are usually introduced. To this end, the amplitude is written in terms of two component spinors $\xi_{t}$ and Pauli matrices $\tau^{k}[8]$,

$$
\begin{aligned}
\mathscr{M} & =8 \pi \sqrt{s} \xi_{t^{\prime}}^{\dagger} \mathcal{F} \xi_{t}, \\
\mathcal{F} & =i \tau \cdot \varepsilon \mathcal{F}_{1}+\tau \cdot \hat{\mathbf{q}} \tau \cdot(\hat{\mathbf{k}} \times \varepsilon) \mathcal{F}_{2}+i \tau \cdot \hat{\mathbf{k}} \hat{\mathbf{q}} \cdot \varepsilon \mathcal{F}_{3}+i \tau \cdot \hat{\mathbf{q}} \hat{\mathbf{q}} \cdot \varepsilon \mathcal{F}_{4} .
\end{aligned}
$$

The hat denotes unit vectors. The $\mathcal{F}_{i}$ are decomposed into electric and magnetic multipoles with the help of derivatives of the Legendre polynomials $P_{l}(z)$ [8],

$$
\begin{aligned}
& \mathcal{F}_{1}=\sum_{l=0}\left[l M_{l+}+E_{l+}\right] P_{l+1}^{\prime}(z)+\left[(l+1) M_{l-}+E_{l-}\right] P_{l-1}^{\prime}(z), \\
& \mathcal{F}_{2}=\sum_{l=1}\left[(l+1) M_{l+}+l M_{l-}\right] P_{l}^{\prime}(z) \\
& \mathcal{F}_{3}=\sum_{l=1}\left[E_{l+}-M_{l+}\right] P_{l+1}^{\prime \prime}(z)+\left[E_{l-}+M_{l-}\right] P_{l-1}^{\prime \prime}(z), \\
& \mathcal{F}_{4}=\sum_{l=1}\left[M_{l+}-E_{l+}-M_{l-}-E_{l-}\right] P_{l}^{\prime \prime}(z)
\end{aligned}
$$

The discussion is restrained to the center of mass frame in the rest of the article. 


\section{Nonrelativistic framework}

To describe the behavior of the multipoles close to threshold - where the energy of the produced pion and of the proton are small - a nonrelativistic calculation is justified. Furthermore, it offers the advantage that all the masses can be set to their physical value. Therefore, all the poles and branch points appear at the correct place in the Mandelstam plane. Moreover, the interaction of the nucleon and the pion is described by effective range parameters, which allows one to directly access the pion-nucleon scattering lengths.

The covariant formulation of nonrelativistic field theories introduced in Refs. [2-4] is used here since it incorporates the correct relativistic dispersion law for the particles. The nonrelativistic proton, neutron and pion fields are denoted by $\psi, \chi$ and $\pi_{k}$, respectively. The kinetic part of the Lagrangian after minimal substitution takes the form (see Ref. [9])

$$
\begin{aligned}
\mathscr{L}_{\text {kin }} & =\sum_{ \pm}\left(i \pi_{ \pm}^{\dagger} D_{t} \mathcal{W}_{ \pm} \pi_{ \pm}-i\left(D_{t} \mathcal{W}_{ \pm} \pi_{ \pm}\right)^{\dagger} \pi_{ \pm}-2 \pi_{ \pm}^{\dagger} \mathcal{W}_{ \pm}^{2} \pi_{ \pm}\right) \\
& +i \psi^{\dagger} D_{t} \mathcal{W}_{p} \psi-i\left(D_{t} \mathcal{W}_{p} \psi\right)^{\dagger} \psi-2 \psi^{\dagger} \mathcal{W}_{p}^{2} \psi \\
& +2 \chi^{\dagger} W_{n}\left(i \partial_{t}-W_{n}\right) \chi+2 \pi_{0}^{\dagger} W_{0}\left(i \partial_{t}-W_{0}\right) \pi_{0}
\end{aligned}
$$

with

$$
\begin{aligned}
& W_{0}=\sqrt{M_{\pi^{0}}^{2}-\triangle}, \\
& W_{n}=\sqrt{m_{n}^{2}-\triangle}, \\
& D_{t} \pi_{ \pm}=\left(\partial_{t} \mp i e A_{0}\right) \pi_{ \pm}, \\
& D_{t} \psi=\left(\partial_{t}-i e A_{0}\right) \psi, \\
& \mathcal{W}_{ \pm}=\sqrt{M_{\pi}^{2}-\mathbf{D}^{2}} \text {, } \\
& \mathcal{W}_{p}=\sqrt{m_{p}^{2}-\mathbf{D}^{2}}, \\
& \mathbf{D} \pi_{ \pm}=(\nabla \pm i e \mathbf{A}) \pi_{ \pm}, \\
& \mathbf{D} \psi=(\nabla+i e \mathbf{A}) \psi \text {. }
\end{aligned}
$$

Note that since the photon is treated as an external field, its kinetic term is absent.

\section{Power counting}

Close to threshold, the momenta of the incoming proton and photon are of the order of the pion mass whereas the outgoing particles have very small momenta. Therefore, we count momenta of the outgoing pion and the outgoing proton as a small quantity of $O(\varepsilon)$ and the momenta of the incoming proton and of the photon as $O(1)$. All the masses are counted as $O(1)$. The mass differences of the charged and neutral pion, $\Delta_{\pi} \equiv M_{\pi}^{2}-M_{\pi^{0}}^{2}$ and of the proton and the neutron, $\Delta_{N} \equiv m_{n}^{2}-m_{p}^{2}$ are counted as $O\left(\varepsilon^{2}\right)$. At first sight, this counting scheme seems to lead to infinitely many terms already in the leading order $p+\gamma \rightarrow p+\pi^{0}$ Lagrangian $\mathscr{L}_{\gamma}$ because derivatives on the incoming fields are not suppressed. However, since the the modulus of the momentum of the incoming particles, $|\mathbf{k}|$, can be expanded in the small momentum $|\mathbf{q}|$,

$$
|\mathbf{k}|=\sum_{n} k_{n} \mathbf{q}^{2 n}, \quad k_{0}=\frac{M_{\pi^{0}}}{2} \frac{2+y}{1+y}, \quad k_{1}=\frac{y^{2}+2 y+2}{4 M_{\pi^{0}}(1+y)}, \quad y=\frac{M_{\pi^{0}}}{m_{p}}
$$

one obtains a valid power counting scheme. The Feynman rule in momentum space of every operator of order $\varepsilon^{0}$ with a given arbitrary number of derivatives can be expanded in powers of the small momentum $\mathbf{q}$, yielding one term of order $\varepsilon^{0}$ without any momenta of the incoming fields 
present and subsequent higher order terms. Doing this for every operator of $O\left(\varepsilon^{0}\right)$, all the resulting leading order terms without any momentum dependence can be described by one operator of order $O\left(\varepsilon^{0}\right)$ in the interaction Lagrangian. The same procedure leads to finite numbers of operators at any given higher order in $\varepsilon$. The derivatives on the incoming fields are only needed to generate unit vectors in the direction of the incoming photon. This shows that the nonrelativistic theory is not capable of predicting the dependence on $|\mathbf{k}|$ even at threshold.

An additional generic parameter $a$ is introduced to count the pion-nucleon scattering vertices. Every pion-nucleon interaction vertex counts as a quantity of order $O(a)$ since the coupling constants are proportional to the pion-nucleon scattering threshold parameters, which are small. The perturbative expansion is therefore a combined expansion in $\varepsilon$ and $a$.

\section{Interaction Lagrangian}

The Lagrangian needed for the calculation of the amplitudes for pion photoproduction reads $\mathscr{L}=\mathscr{L}_{\text {kin }}+\mathscr{L}_{\gamma}+\mathscr{L}_{\pi N}$, where $\mathscr{L}_{\text {kin }}$ denotes the kinetic part, $\mathscr{L}_{\gamma}$ incorporates the interaction with the photon field and and $\mathscr{L}_{\pi N}$ describes the pion-nucleon sector.

In the pion nucleon sector, the leading terms of the Lagrangian have been given before in Ref. [10]. First, some notation is introduced in order to write the Lagrangian in a compact form. For every channel $n$, we collect the charges of the outgoing and the incoming pions in the variables $v$ and $w,(n ; v, w):(0 ; 0,0),(1 ; 0,+),(2 ;+,+),(3 ; 0,0),(4 ;-, 0),(5 ;-,-)$, thereby assigning unique values to the variables $v$ and $w$ once $n$ is given. The Lagrangian reads

$$
\begin{aligned}
\mathscr{L}_{\pi N} & =\left(\begin{array}{ll}
\psi^{\dagger} & \chi^{\dagger}
\end{array}\right)\left(\begin{array}{cc}
T_{\{0,5\}} & T_{\{1,4\}} \\
T_{\{1,4\}}^{\dagger} & T_{\{2,3\}}
\end{array}\right)\left(\begin{array}{c}
\psi \\
\chi
\end{array}\right), \\
T_{\mathscr{C}} & =\sum_{n \in \mathscr{C}}\left[C_{n} \pi_{v}^{\dagger} \pi_{w}+D_{n}^{(1)} \nabla^{k} \pi_{v}^{\dagger} \nabla^{k} \pi_{w}+D_{n}^{(2)} \pi_{v}^{\dagger} \overleftrightarrow{\triangle} \pi_{w}+i D_{n}^{(3)} \tau^{k} \varepsilon^{i j k} \nabla^{i} \pi_{v}^{\dagger} \nabla^{j} \pi_{w}\right]
\end{aligned}
$$

with the abbreviation $f \overleftrightarrow{\triangle} g \equiv f \triangle g+(\triangle f) g$.

For $\mathscr{L}_{\gamma}$, the photon is treated as an external vector field A which is odd under parity and timereversal transformations. One obtains for the gauge invariant Lagrangian

$$
\begin{aligned}
\mathscr{L}_{\gamma}^{(0)} & =-i G_{0}^{(1)} \psi^{\dagger} \tau^{k} \psi E^{k} \pi_{0}^{\dagger}, \\
\mathscr{L}_{\gamma}^{(1)} & =-i G_{1}^{(2)} \psi^{\dagger} \tau^{k} \psi \nabla^{j} E^{k} \nabla^{j} \pi_{0}^{\dagger}+i G_{2}^{(1)} \psi^{\dagger} \tau^{m} \tau^{l} \psi B^{l} \nabla^{m} \pi_{0}^{\dagger} \\
& -i G_{3}^{(2)} \psi^{\dagger} \tau^{j} \psi \nabla^{j} E^{k} \nabla^{k} \pi_{0}^{\dagger}, \\
\mathscr{L}_{\gamma}^{(2)} & =-i G_{4}^{(3)} \psi^{\dagger} \tau^{k} \psi \nabla^{j l} E^{k} \nabla^{j l} \pi_{0}^{\dagger}-i G_{5}^{(1)} \psi^{\dagger} \tau^{k} \psi E^{k} \triangle \pi_{0}^{\dagger} \\
& +i G_{6}^{(2)} \psi^{\dagger} \tau^{m} \tau^{l} \psi \nabla^{n} B^{l} \nabla^{m n} \pi_{0}^{\dagger}-i G_{7}^{(3)} \psi^{\dagger} \tau^{j} \psi \nabla^{j l} E^{k} \nabla^{k l} \pi_{0}^{\dagger} \\
& -i G_{8}^{(1)} \psi^{\dagger} \tau^{j} \psi E^{k} \nabla^{j k} \pi_{0}^{\dagger}, \\
\mathscr{L}_{\gamma}^{(3)} & =-i G_{9}^{(2)} \psi^{\dagger} \tau^{k} \psi \nabla^{j} E^{k} \triangle \nabla^{j} \pi_{0}^{\dagger}-i G_{10}^{(4)} \psi^{\dagger} \tau^{k} \psi \nabla^{l m n} E^{k} \nabla^{l m n} \pi_{0}^{\dagger} \\
& +i G_{11}^{(1)} \psi^{\dagger} \tau^{m} \tau^{l} \psi B^{l} \triangle \nabla^{m} \pi_{0}^{\dagger}+i G_{12}^{(3)} \psi^{\dagger} \tau^{m} \tau^{l} \psi \nabla^{i n} B^{l} \nabla^{m i n} \pi_{0}^{\dagger} \\
& -i G_{13}^{(2)} \psi^{\dagger} \tau^{j} \psi \nabla^{j} E^{k} \triangle \nabla^{k} \pi_{0}^{\dagger}-i G_{14}^{(4)} \psi^{\dagger} \tau^{j} \psi \nabla^{j l m} E^{k} \nabla^{k l m} \pi_{0}^{\dagger} \\
& -i G_{15}^{(2)} \psi^{\dagger} \tau^{j} \psi \nabla^{l} E^{k} \nabla^{j k l} \pi_{0}^{\dagger} .
\end{aligned}
$$


The upper index on the coupling constants is introduced for later convenience. Here, the notation $\nabla^{i_{1} i_{2} \ldots i_{k}} \equiv \nabla^{i_{1}} \nabla^{i_{2}} \ldots \nabla^{i_{k}}$ is used. Since the structure of the Lagrangian for the other required channel $p \gamma \rightarrow n \pi^{+}$stays the same, one only has to replace the coupling constants and the field operators, $\left\{\psi^{\dagger}, \pi_{0}^{\dagger}, G_{i}^{(n)}\right\} \rightarrow\left\{\chi^{\dagger}, \pi_{+}^{\dagger}, H_{i}^{(n)}\right\}$. The full interaction Lagrangian $\mathscr{L}_{\gamma}$ is then given by adding the $\mathscr{L}_{\gamma}^{(i)}$ of both channels.

\section{Matching relations}

In the pion-nucleon sector, the coupling constants of the nonrelativistic Lagrangian, $C_{i}$ and $D_{i}^{(k)}$ can be expressed in terms of pion-nucleon scattering lengths of the $S$-wave and $P$-wave, $a_{0+}$ and $a_{1 \pm}$ and effective range parameters $b_{0+}$, respectively. Adopting the notation of Ref. [11], in the isospin limit, the isospin decomposition of the $\pi N$ scattering amplitudes reads

$$
\begin{aligned}
T_{p \pi^{0} \rightarrow p \pi^{0}} & =T_{n \pi^{0} \rightarrow n \pi^{0}}=T^{+}, & T_{p \pi^{0} \rightarrow n \pi^{+}}=T_{n \pi^{0} \rightarrow p \pi^{-}}=-\sqrt{2} T^{-}, \\
T_{n \pi^{+} \rightarrow n \pi^{+}} & =T_{p \pi^{-} \rightarrow p \pi^{-}}=T^{+}+T^{-} . &
\end{aligned}
$$

Defining $\mathscr{N}=4 \pi\left(m_{p}+M_{\pi}\right)$, one finds

$$
\begin{array}{lll}
C_{0}=2 \mathscr{N} a_{0+}^{+}, & C_{1}=2 \sqrt{2} \mathscr{N} a_{0+}^{-}, & C_{2}=2 \mathscr{N}\left(a_{0+}^{+}+a_{0+}^{-}\right), \\
C_{3}=C_{0}, & C_{4}=C_{1}, & C_{5}=C_{2} .
\end{array}
$$

The matching conditions for the $D_{i}^{(k)}$ are given in a generic form only. The isospin index of the threshold parameters can be inferred from Eq. $(6.1)^{1}$.

$$
\begin{array}{ll}
D_{i}^{(1)}=2 \mathscr{N}\left(2 a_{1+}+a_{1-}\right), & D_{i}^{(2)}=-\mathscr{N}\left(\frac{a_{0+}}{2 m_{p} M_{\pi}}+b_{0+}\right), \\
D_{i}^{(3)} & =2 \mathscr{N}\left(a_{1-}-a_{1+}\right) .
\end{array}
$$

Here, higher order terms in the threshold parameters have been dropped. The corrections to these relations which appear due to isospin breaking have to be calculated within the underlying relativistic theory. For the $C_{i}$, they can be found in Refs. [12-15]. Note that the second line in Eq. (6.2) is only true in the isospin limit.

The constants $G_{i}^{(n)}$ and $H_{i}^{(n)}$ on the other hand are related to the threshold parameters of the electric and magnetic multipoles of the pertinent channel. In the isospin limit, the expansion of the real part of the multipole $X_{l \pm}$ close to threshold is written in the form

$$
\operatorname{Re} X_{l \pm}(s)=\sum_{k=0}^{\infty} \bar{X}_{l \pm, 2 k}|\mathbf{q}|^{l+2 k}
$$

which defines the threshold parameters $\bar{X}_{l \pm, 2 k}$. In the following, the relations of the coupling constants $G_{i}^{(n)}$ to these threshold parameters is given at leading order in the pion nucleon threshold parameters. Since the nonrelativistic theory is not suited for the study of the dependence of the

\footnotetext{
${ }^{1}$ Note that we use the Condon-Shortley phase convention.
} 
multipoles on $|\mathbf{k}|$, in this analysis, all vectors $\mathbf{k}$ are turned into unit vectors by the pertinent redefinition of the coupling constants,

$$
G_{i}^{(n)}=\mathscr{N}_{0} k_{0}^{-n} G_{i}, \quad \mathscr{N}_{0}=4 \pi\left(m_{p}+M_{\pi^{0}}\right) .
$$

Note that the higher order corrections due to Eq. (4.1) have to be taken care of in the matching relations. Again, these relations pick up isospin breaking corrections which have to be evaluated in the underlying relativistic theory.

Only the matching equations for the couplings of the Lagrangians $\mathscr{L}_{\gamma}^{(0)}$ and $\mathscr{L}_{\gamma}^{(1)}$ are indicated here. The remaining relations can be found in Ref. [5]. To ease notation, $\bar{X}_{i \pm} \equiv \bar{X}_{i \pm, 0}$ is used.

$$
\begin{array}{ll}
G_{0}=2 \bar{E}_{0+}, & G_{1}=6\left(\bar{E}_{+1}+\bar{M}_{+1}\right), \\
G_{2}=-2\left(\bar{M}_{-1}+2 \bar{M}_{+1}\right), & G_{3}=6\left(\bar{E}_{1+}-\bar{M}_{1+}\right) .
\end{array}
$$

For the coupling constants $H_{i}$ the algebraic form of the relations is identical. However, the multipoles of the pertinent channels appear and the masses in Eq. (6.5) have to be adjusted.

All coupling constants are assumed to be real. See Ref. [5] for a discussion of this issue.

\section{Results}

In the following, we provide the expressions for the electric and magnetic multipoles $E_{l+}$ for $l=0,1$ and $M_{l \pm}$ for $l=1$. The result is written in the form

$$
X_{l, \pm}(s)=X_{l \pm}^{\text {tree }}(s)+X_{l \pm}^{1 \mathrm{Loop}}(s)+X_{l \pm}^{2 \mathrm{Loop}}(s) \cdots
$$

where $s=\left(p_{1}+k\right)^{2}$ and the ellipsis denote higher order terms in the expansion in $\varepsilon$ and $a$.

\subsection{Tree-level}

The tree level result can be written in the form $X_{l \pm}^{\text {tree }}(s)=X_{l \pm}^{t} \mathbf{q}^{l}+X_{l \pm, 2}^{t} \mathbf{q}^{2+l}+\cdots$ with the coefficients

$$
\begin{array}{rlrl}
E_{0+}^{t}=G_{0}, & 3 E_{0+, 2}^{t} & =G_{4}-3 G_{5}+G_{6}-G_{8}, \\
6 M_{1+}^{t}=G_{1}-G_{3}, & M_{1+, 2}^{t} & =-\frac{1}{6} G_{9}+\frac{1}{10} G_{10}+\frac{1}{15} G_{12}+\frac{1}{6} G_{13}-\frac{1}{30} G_{14}, \\
3 M_{1-}^{t}=G_{3}-G_{1}-3 G_{2}, & M_{1-, 2}^{t} & =\frac{1}{3} G_{9}-\frac{1}{5} G_{10}+G_{11}-\frac{1}{3} G_{12} \\
& -\frac{1}{3} G_{13}+\frac{1}{15} G_{14}, \\
6 E_{1+}^{t}=G_{1}+G_{3}, & E_{1+, 2}^{t} & =-\frac{1}{6} G_{9}+\frac{1}{10} G_{10}+\frac{1}{15} G_{12}-\frac{1}{6} G_{13} \\
& +\frac{1}{30} G_{14}-\frac{1}{15} G_{15} .
\end{array}
$$

One observes that $D$-waves appear naturally at order $\varepsilon^{2}$ in this framework (see also Ref. [16]).

\subsection{One-loop}

All the one-loop contributions are proportional to the basic integral

$$
J_{a b}\left(P^{2}\right)=\int \frac{d^{D} l}{i(2 \pi)^{D}} \frac{1}{2 \omega_{a}(\mathbf{I}) 2 \omega_{b}(\mathbf{P}-\mathbf{l})} \frac{1}{\left(\omega_{a}(\mathbf{l})-l_{0}\right)\left(\omega_{b}(\mathbf{P}-\mathbf{l})-P_{0}+l_{0}\right)},
$$



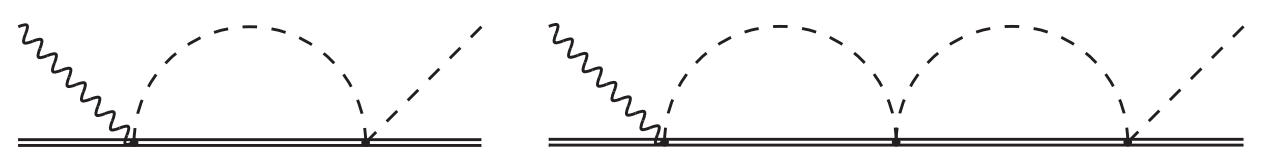

Figure 1: One- and two loop topologies needed to calculate the amplitude. The double line generically denotes a nucleon, the dashed line a pion and the wiggly line indicates the external electromagnetic field.

$$
\begin{aligned}
& \omega_{ \pm}(\mathbf{p})=\sqrt{M_{\pi}^{2}+\mathbf{p}^{2}}, \quad \omega_{i}(\mathbf{p})=\sqrt{m_{i}^{2}+\mathbf{p}^{2}}, \quad i=n, p \\
& \omega_{0}(\mathbf{p})=\sqrt{M_{\pi^{0}}^{2}+\mathbf{p}^{2}}, \quad P^{2}=P_{0}^{2}-\mathbf{P}^{2} .
\end{aligned}
$$

In the limit $D \rightarrow 4$,

$$
J_{a b}\left(P^{2}\right)=\frac{i}{16 \pi s} \sqrt{\left(s-\left(m_{a}+M_{\pi^{b}}\right)^{2}\right)\left(s-\left(m_{a}-M_{\pi^{b}}\right)^{2}\right)},
$$

which is a quantity of order $\varepsilon$. The one-loop result up to and including order $O\left(a \varepsilon^{4}\right)$ reads

$$
\left(\begin{array}{c}
E_{0+}^{1 \text { Loop }}(s) \\
\frac{1}{|\mathbf{q}|} M_{1+}^{1 \mathrm{Loop}}(s) \\
\frac{1}{|\mathbf{q}|} M_{1-}^{1 \mathrm{Loop}}(s) \\
\frac{1}{|\mathbf{q}|} E_{1+}^{1 \mathrm{Loop}}(s)
\end{array}\right)=\left(\begin{array}{cc}
P_{11} & P_{12} \\
P_{21} & P_{22} \\
P_{31} & P_{32} \\
P_{41} & P_{42}
\end{array}\right)\left(\begin{array}{c}
J_{p 0}(s) \\
J_{n+}(s)
\end{array}\right) .
$$

The elements $P_{i k}$ are functions of the pion momentum $\mathbf{q}$ and the coupling constants of the Lagrangian,

$$
\begin{aligned}
P_{11} & =G_{0} C_{0}+\mathbf{q}^{2}\left(C_{0} E_{0+, 2}^{(p 0), t}-2 D_{0}^{(2)} G_{0}\right), \\
P_{12} & =C_{1} H_{0}+h^{2}\left(s, m_{c}, M_{\pi^{d}}\right)\left(C_{1} E_{0+, 2}^{(n+), t}-D_{1}^{(2)} H_{0}\right)-\mathbf{q}^{2} D_{1}^{(2)} H_{0}, \\
18 P_{21} & =\mathbf{q}^{2}\left(D_{0}^{(1)}-D_{0}^{(3)}\right)\left(G_{1}-G_{3}\right), \\
18 P_{22} & =h^{2}\left(s, m_{c}, M_{\pi^{d}}\right)\left(D_{1}^{(1)}-D_{1}^{(3)}\right)\left(H_{1}-H_{3}\right), \\
9 P_{31} & =\mathbf{q}^{2}\left(D_{0}^{(1)}+2 D_{0}^{(3)}\right)\left(G_{3}-G_{1}-3 G_{2}\right), \\
9 P_{32} & =h^{2}\left(s, m_{c}, M_{\pi^{d}}\right)\left(D_{1}^{(1)}+2 D_{1}^{(3)}\right)\left(H_{3}-H_{1}-3 H_{2}\right), \\
18 P_{41} & =\mathbf{q}^{2}\left(D_{0}^{(1)}-D_{0}^{(3)}\right)\left(G_{1}+G_{3}\right), \\
18 P_{42} & =h^{2}\left(s, m_{c}, M_{\pi^{d}}\right)\left(D_{1}^{(1)}-D_{1}^{(3)}\right)\left(H_{1}+H_{3}\right),
\end{aligned}
$$

where $E_{0+, 2}^{(c), t}$ denotes the pertinent coefficient of the tree level result of channel (c), see Eq. (7.2), and $h^{2}\left(s, m_{c}, M_{\pi^{d}}\right)$ is given by

$$
h^{2}\left(s, m_{c}, M_{\pi^{d}}\right)=\frac{\left(s-\left(m_{n}+M_{\pi^{+}}\right)^{2}\right)\left(s-\left(m_{n}-M_{\pi^{+}}\right)^{2}\right)}{4 s},
$$

which is a quantity of order $\varepsilon^{2}$. Eq. (7.5) and (7.6) clearly show the advantage of the nonrelativistic description: The strength of the cusp at leading order is parameterized in terms of the coupling constant $C_{1}$ and the ratio $H_{0} / G_{0}$. 


\section{Phase of $\pi^{0} p \rightarrow \pi^{0} p$ scattering}

In an isospin symmetric world, the phase of the multipole $E_{0+}$ is directly related to the phase shift of the $S$-wave of pion-nucleon scattering by virtue of the Fermi-Watson theorem [17]. In Ref. [7], it is shown with a coupled channel $S$-matrix approach that to leading order in $e$, below the $\pi^{+} n$ threshold, the phase of the $S$ wave of $\pi^{0} p \rightarrow \pi^{0} p$ scattering is equal to the phase of $E_{0+}$,

$$
\tan \delta_{p \pi^{0} \rightarrow p \pi^{0}}=\tan \frac{\operatorname{Im} E_{0+}}{\operatorname{Re} E_{0+}} \equiv \tan \delta_{E_{0+}} .
$$

The framework developed here allows one to test this statement order by order in the perturbative expansion. To this end, the phase of $E_{0+}$ below the second threshold is calculated up to and including $O\left(a^{2} \varepsilon^{4}\right)$

$$
\begin{aligned}
\tan \delta_{E_{0+}} & =C_{0} \operatorname{Im} J_{p 0}+C_{1}^{2} J_{n+} \operatorname{Im} J_{p 0}-2 D_{0}^{(2)} \operatorname{Im} J_{p 0} \mathbf{q}^{2} \\
& -2 C_{1} D_{1}^{(2)} J_{n+} \operatorname{Im} J_{p 0} \mathbf{q}^{2}-2 C_{1} D_{1}^{(2)} J_{n+} \operatorname{Im} J_{p 0} h^{2}\left(s, m_{n}, M_{\pi}\right)+\cdots
\end{aligned}
$$

Calculating $\pi^{0} p \rightarrow \pi^{0} p$ scattering to the same order with the Lagrangian given in Eq. (5.1), one finds that the phase of the $S$-wave below the second threshold is indeed equal to Eq. (8.2). However, the main object of interest is the phase of the $S$-wave of $\pi^{0} p \rightarrow \pi^{0} p$ scattering in the isospin symmetry limit,

$$
\tan \bar{\delta}_{p \pi^{0} \rightarrow p \pi^{0}}=C_{0} \operatorname{Im} J_{p 0}+\frac{C_{1}^{2}}{C_{0}} \operatorname{Im} J_{n+} \simeq a_{0+}^{+} \mathbf{q}+2 \frac{a_{0+}^{-2}}{a_{0+}^{+}} \mathbf{q}+\cdots,
$$

which does not agree with $\delta_{p \pi^{0} \rightarrow p \pi^{0}}$ in the presence of isospin violations already at leading order.

\section{Summary and conclusion}

We study the photoproduction reaction of pions on the nucleon using a nonrelativistic framework. The electric and magnetic multipoles $E_{l+}$ for $l=0,1$ and $M_{1 \pm}$ are calculated in a systematic double expansion in the final state pion- and nucleon momenta (counted as a small quantity of order $\varepsilon$ ) and the threshold parameters of $\pi N$ scattering (denoted by $a$ ). Explicit representations for the multipoles up to and including $\varepsilon^{3}$ and $\varepsilon^{4} a$ are provided. The corresponding two-loop results as well as a expressions for the multipole amplitudes in the remaining three reaction channels can be found in Ref. [5].

The representation is valid in the low energy region, at least up to a photon energy in the lab frame of $E_{\gamma}=165 \mathrm{MeV}$. It accurately describes the cusp structure and allows one to determine the pion-nucleon threshold parameters from experimental data.

The relation of the phase of the electric multipole $E_{0+}$ in the $(p 0)$ channel to the phase of the $S$-wave of $\pi^{0} p \rightarrow \pi^{0} p$ scattering is discussed in the presence of isospin violation. A relation found in earlier work [7] is confirmed. We stress that the relation does not allow one to obtain the phase of $\pi^{0} p \rightarrow \pi^{0} p$ scattering in the isospin limit.

Acknowledgements. I would like to thank the organizers for a very interesting workshop. I am also indebted to J. Gasser, B. Kubis, A. Manohar and U.-G. Meißner for informative discussions and B. Kubis for comments on the manuscript. This work was supported in part by the Department of Energy under Grant DE-FG03-97ER40546 and by the Swiss National Science Foundation. 


\section{References}

[1] A. Schmidt et al., Phys. Rev. Lett. 87 (2001) 232501 [arXiv:nucl-ex/0105010].

[2] G. Colangelo, J. Gasser, B. Kubis and A. Rusetsky, Phys. Lett. B 638 (2006) 187 [arXiv:hep-ph/0604084].

[3] M. Bissegger, A. Fuhrer, J. Gasser, B. Kubis and A. Rusetsky, Phys. Lett. B 659 (2008) 576 [arXiv:0710.4456 [hep-ph]].

[4] B. Kubis and S. P. Schneider, Eur. Phys. J. C 62 (2009) 511 [arXiv:0904.1320 [hep-ph]].

[5] A. Fuhrer, arXiv:0909.3121 [hep-ph].

[6] V. Bernard, N. Kaiser and U. G. Meissner, Z. Phys. C 70 (1996) 483 [arXiv:hep-ph/9411287].

[7] A. M. Bernstein, Phys. Lett. B 442 (1998) 20 [arXiv:hep-ph/9810376].

[8] G. F. Chew, M. L. Goldberger, F. E. Low and Y. Nambu, Phys. Rev. 106 (1957) 1345.

[9] M. Bissegger, A. Fuhrer, J. Gasser, B. Kubis and A. Rusetsky, Nucl. Phys. B 806 (2009) 178 [arXiv:0807.0515 [hep-ph]].

[10] V. E. Lyubovitskij and A. Rusetsky, Phys. Lett. B 494 (2000) 9 [arXiv:hep-ph/0009206].

[11] G. Höhler, in Landolt-Börnstein, 9b2, ed. H. Schopper (Springer, Berlin, 1983).

[12] J. Gasser, M. A. Ivanov, E. Lipartia, M. Mojzis and A. Rusetsky, Eur. Phys. J. C 26 (2002) 13 [arXiv:hep-ph/0206068].

[13] U. G. Meissner, U. Raha and A. Rusetsky, Phys. Lett. B 639 (2006) 478 [arXiv:nucl-th/0512035].

[14] M. Hoferichter, B. Kubis and U. G. Meissner, Phys. Lett. B 678 (2009) 65 [arXiv:0903.3890 [hep-ph]].

[15] M. Hoferichter, B. Kubis and U. G. Meißner, arXiv:0909.4390 [hep-ph].

[16] C. Fernandez-Ramirez, A. M. Bernstein and T. W. Donnelly, arXiv:0907.3463 [nucl-th].

[17] E. Fermi, Suppl. Nuovo Cimento 2 (1955) 58, K. M. Watson, Phys. Rev. 95 (1954) 228. 